\title{
Social Learning, Environmental Adversity and the Evolution of Cooperation
}

\author{
Peter Andras ${ }^{1}$ \\ ${ }^{1}$ School of Computing and Mathematics \\ Keele University \\ Newcastle-under-Lyme, Staffordshire, ST5 5BG, UK \\ p.andras@keele.ac.uk
}

\begin{abstract}
Social learning plays a key role in the evolution of cooperation in humans and other animals. It has also been shown both theoretically and experimentally that environmental adversity is also a key determinant of the evolution of cooperation among individuals. Here we investigate the impact of social learning on the evolution of cooperation in the context of a range of levels of environmental adversity. We used an agent-based simulated world of asexual individuals that communicate and play a probabilistic version of the Prisoner's Dilemma game. We considered simulated worlds either with or without random spreading of the offspring and two variants of social learning, either copying to some extent all communication rules or copying fully some of the communication rules of the best performing neighbor individual. The results show that in the case of spreading of the offspring, social learning increases the level of cooperation and reverses the association between this and the level of environmental adversity, i.e. low adversity with social learning implies higher level of cooperation. Copying fully some communication rules also increases the steady-state level of communication complexity in the simulated agent communities. The results suggest that the level of cooperation in communities of individuals may get boosted alternately by highly adverse environments and by layers of social learning in low adversity environments.
\end{abstract}

\section{Introduction}

The emergence and evolution of cooperation among individual humans and animals is a fundamental question of social evolution (Axelrod, 1997). In general it is assumed that cooperation emerges either because of kin selection, or direct or indirect reciprocation, or because of some form of social clustering or due to the group level selection of groups with more cooperating individuals (Rand and Nowak, 2013). It has been also shown that social factors, such as enforcement of rule following, also contribute significantly to the evolution of cooperation (Sigmund et al, 2010).

An external factor that has critical influence on the level of cooperation is environmental adversity, which includes both the harshness of the environment (i.e. scarcity of resources) and the variability of the environment (i.e. the variability and extent of the lack of predictability of the level of available resources) (Andras et al, 2007; Andras et al. 2003). Theoretical, simulation and real world experimental results confirm that in general higher level of environmental adversity implies higher level of cooperation in communities of individuals existing in the presence of such environmental constraints (Krams et al, 2010; Spinks et al, 2000; Rand et al, 2014; Potts and Faith, 2015).

Social learning in general means that individuals within a community adapt their behavior such that they follow behavioral patterns of other individuals (Flinn, 1997). The other individual may be chosen on various grounds, for example it can be the most successful individual in some predefined sense or it can be the oldest neighboring individual. It has been suggested that social learning supports cooperation in communities of individuals, especially in the context of humans (Boyd and Richerson, 2009), however, there are also claims of the opposite effect in the relevant literature (Heyes, 2013).

The level of cooperation can be measured directly in experiments (both in the case of agent-based simulations and in the real world). Having additional measures of correlates of cooperation is useful to understand better the context of the measured level of cooperation. One such measure is the communication complexity of the interactions between the individuals of the community (Andras, 2008). Communication complexity decreases with increased environmental adversity and this contributes to the increase in the level of cooperation (Andras, 2008).

Here we present the use of an agent-based simulation which implements communicating agents that play prisoner's dilemma games (Axelrod, 1997) to investigate the role of social learning in the context of evolution of cooperation in communities of selfish individuals. We considered two variants of the simulated world, one where the offspring of the asexual individuals do not get dispersed widely from the location of their parent, and another where the offspring is dispersed away from the location of the parent - in general the simulation without dispersal of the offspring have higher levels of cooperation at the steady-state level than the simulation with offspring dispersal (Andras et al, 2003; Andras et al, 2006). We also considered two variants of social learning, in one case the learner copies to some extent the communication rules of the most successful neighbor, in the other case the learner copies fully some of the communication rules of the most successful neighbor. The results show that copying fully some communication rules increases the level of cooperation more than the considered alternative social learning method. Both social learning methods have much more effect if the offspring are widely dispersed. Social 
learning with full copying of some communication rules also reverses the association between environmental adversity and the level of cooperation, making the level of cooperation increase with the decrease of the environmental adversity. In addition, social learning with full copying of some communication rules leads to higher communication complexity in the simulated agent communities than the use of the other social learning method or simulations without any social learning. The results suggest that the level of cooperation in communities of individuals may get boosted alternately by highly adverse environments and by layers of social learning in low adversity environments.

The rest of the paper is structured as follows. First we review briefly the relevant results from the literature. Next we provide a description of the agent-based simulation that we used. This is followed by the presentation of the detailed results. Finally the paper is closed by the discussion and conclusions section.

\section{Background}

There are several theories about the mechanisms behind the emergence and evolution of cooperation in communities of selfish individuals. Kin selection assumes that related individuals recognize each other on the basis of their similarity and the likelihood of their cooperation with their kin is high in order to support the success and spreading of the kin (Rand and Nowak, 2013). Direct reciprocity assumes that individuals are likely to reciprocate the cooperative help received from others and expect further reciprocation of cooperation by others who benefit from this (Rand and Nowak, 2013). Indirect reciprocity relies on the assumption that individuals observe the behavior of other individuals and they are more likely to cooperate with those who are seen to cooperate with others (Rand and Nowak, 2013). Group selection based mechanisms assume that individuals belonging to groups characterized by higher level of cooperation are more likely to survive and have offspring because their group has a better chance of survival as a group due to the benefits from the high level of cooperation within the group (Rand and Nowak, 2013). Other models rely on emergent population structure (e.g. spatial constraints) that drive cooperators together and exclude non-cooperators, giving in such way an advantage to the emergent communities of cooperators over other emergent communities not dominated by cooperators (Rand and Nowak, 2013).

Environmental adversity is an important determinant of the emergence and evolution of cooperation (Andras et al, 2007; Andras et al, 2003). Theoretical analysis shows that higher environmental adversity (harsher environment or more variable environment) implies higher level of cooperation among individuals in communities that survive in high adversity environment (Andras et al, 2007; Andras et al, 2003). Experimental results about a range of animals, humans and agent-based simulation results confirm this theoretical result, showing that indeed, exposure to higher predation risk or higher variability of environmental resources or risks lead to higher frequency of cooperative behavior between individuals (Krams et al, 2010; Spinks et al, 2000; Rand et al, 2014).
Theoretical and agent-based simulation analysis of the environmental risk conceptualized as the variance of the available resources shows that the experienced subjective risk is always bigger than the objective risk and the difference is bigger in harsher environments (Andras et al, 2007). It has been also shown that the effective risk after taking into account the effect of cooperation is always smaller than the subjective risk and that the effective risk is practically stable across a range of subjective risk levels and this stable effective risk is slightly increasing with the harshness of the environment (Andras et al, 2006). This implies that higher subjective risk perceived by individuals triggers more cooperation in order to bring down the level of the effective risk to the stable level of this.

In the context of communicating individuals who negotiate before making the decision about cooperation the complexity of the communication language that they use contributes to the overall environmental risk. It has been shown through agent-based simulation studies that indeed communication complexity is lower in the case of higher external environmental risk (Andras, 2008). Note that the language complexity is measured in terms of the variability of the communication rules and not as the length of communication sequences preceding the decision on cooperation. This result implies that the communication complexity measure is a useful correlate of the extent of reduction of the effective risk through reduction of the unreliability of communications between individuals.

Social learning plays a key role in organizing the social role of individuals in the context of their social environment provided by their community (Flinn, 1997). The essence of social learning is the copying or imitating the behavior of one individual by another individual. There are several mechanisms of social learning, some being context-dependent others being content-dependent, some are oriented towards specific individuals (e.g. richest, most successful, oldest, most similar) others are driven by frequency of behaviors (e.g. most frequent is copied) or by the state of individuals (e.g. experiencing high dissatisfaction) (Rendell et al, 2010). Social learning may work by copying a fully or partially a whole sequence of consecutive behaviors or by aiming to emulate the outcome of a sequence of behaviors, or by some intermediate variant of behavioral copying (Rendell et al, 2010). Social learning may also be supported by enforcement of rules in various forms of punishment applied to individuals who do not conform to the rules (Sigmund et al, 2010).

Agent-based simulations have been used to study various aspects of social learning (e.g. choice of social learning mechanisms) (Nakahashi et al, 2012; Seltzer and Smirnov, 2015; Molleman et al, 2013). Such simulations usually implement a small range of alternative social learning mechanisms and analyze their impact on the behavior of the simulated agent community.

The role of social learning in the context of emergence and evolution of cooperation has been considered in a number of settings. In general it is suggested that social learning is a key contributor to the evolution of cooperation among humans and possibly also among other animals (Boyd and Richerson, 2009; Rendell et al, 2010; Chudek et al, 2013) It has been shown that in simulated social networks imitation of socially distant individuals increase the level of cooperation within the 
agent community (Seltzer and Smirnov, 2015). Other agentbased simulation studies show that certain forms of social learning (e.g. conformism) reduces the level of cooperation in simulated communities (Molleman et al, 2013; Burton et al, 2015). There are also more theoretical / conceptual investigations that question the level of contribution of social learning to the emergence and evolution of cooperation among humans (Heyes, 2013).

\section{Simulated Agent Communities}

The simulated world of agents is placed in a two dimensional space arranged as a torus in both dimensions and having the size of 1000 in both dimensions. The agents move randomly in this space in each turn (up to 5 units in both dimensions).

Each simulation runs for 400 time turns. In each turn each agent picks randomly another agent from its spatial neighborhood to interact with. An agent is allowed to interact with only one other agent at any time and some agent may stay without interaction partner in some of the time turns.

The agents own resources and they spend these to survive. If the resource amount of an agent goes below zero the agent dies. The agents use their current level of resources to set their level of resources in the next turn. They may also play a resource generation game with their interaction partner.

The agents interact using a communication language consisting of the symbols: ' 0 ','s', 'i', 'y','n','h' and 't'. The meaning of the communication symbols are as follows: ' 0 ' no intention of communication, 's' - start of communication, 'i' - maintaining the communication, ' $y$ ' - indication of the willingness to engage into resource sharing, ' $n$ ' - indication of no further interest in communication, ' $h$ ' - effective sharing of the resources, ' $t$ ' - not sharing the resources after an indication of willingness to engage into sharing. The last two symbols, ' $h$ ' and ' $t$ ' effectively mean the resource-sharing or no-resource-sharing actions of the agents. The generation of communication symbols by agents is determined by probabilistic communication rules of the agents. These rules are expressed as follows

$$
\underset{U_{\text {curren }}, U_{\text {current }}^{\prime} \rightarrow \rightarrow_{p 1} U_{\text {new }, 1} ;: \rightarrow{ }_{p 2} U_{\text {new }, 2} ; \ldots ; \rightarrow{ }_{p k} U_{\text {new }, k}}{ }
$$

where $U_{\text {current }}$ is the current communication symbol produced by the agent, $U_{\text {current }}$ is the current communication symbol produced by the communication partner agent, $U_{\text {new }, j}$ is the j-th possible communication symbol that may be produced by the agent following the previous production of the symbol $U_{\text {current }}$ and the production of the symbol $U_{\text {current }}^{\prime}$ by the communication partner agent, and $p_{i}$ is the probability of producing $U_{\text {new,i }}$ the symbol. Naturally we have that $p_{1}+$ $p_{2}+\ldots+p_{k}=1$. For example, a communication rule can be the following

$$
L:
$$

$$
i, i, \rightarrow_{0.5} i ; \rightarrow_{0.2} y ; \rightarrow_{0.3} n
$$

which means that after producing the symbol ' $i$ ' and receiving the symbol ' $i$ ' from the communication partner, with 0.5 probability the agent will produce the symbol 'i', with 0.2 probability the symbol ' $y$ ' and with 0.3 probability the symbol 'n'.
An example of a sequence of communications between two agents is: ' $\mathrm{s}_{1}, \mathrm{~s}_{2}, \mathrm{i}_{1}, \mathrm{i}_{2}, \mathrm{i}_{1}, \mathrm{i}_{2}, \mathrm{y}_{1}, \mathrm{i}_{2}, \mathrm{y}_{1}, \mathrm{n}_{2}$ ', where the indices are the identifiers of the two agents. If the communication process between two agents carries on for too long without reaching the production of the action symbols ' $\mathrm{t}$ ' or ' $\mathrm{h}$ ' (the length limit was set to 20 symbols), the communication terminates as it is considered too long for the time turn. The communication between two agents may also terminate if either of them starts by producing the ' 0 ' symbol, if one of them produces the ' $n$ ' symbol, or if they both produce a ' $t$ ' or ' $h$ ' symbol. In the latter case the agents engage in a prisoners' dilemma game where the outcome of the game depends on the actions of the involved agents, i.e. they cooperate if both of them produce the symbol ' $h$ ' otherwise one of them or both of them tries to cheat (by producing the symbol ' $t$ ').

When the agents enter the playing of the prisoners' dilemma game they jointly invest their available resources to generate new resources. The overall payoff of the game is the difference between the sum of the amounts of new resources that each agent would have without entering the game and the amount of resources that can be generated by using the combined current resources of the agents. If an agent cheats while the interaction partner is willing to cooperate the cheating agent takes the full payoff and the other agent gets no extra resources in addition to what it can generate by itself with its own available resources. If they both decide to cooperate they share the full payoff equally and this gets added to the amount of resources that they would generate individually. If both agents decide to try to cheat no extra resource is allocated to either of the agents.

The generation of effective new resources is realized in a probabilistic manner. The actual value is picked from a uniform distribution where the mean value of the distribution is given by the calculated value of new resources and the halfwidth (equivalent of variance) of the distribution is given by the environmental risk level $(\sigma)$ that characterizes the simulated world of the agents. Low environmental risk (low variance) means than the actual value of the new resource is close to the calculated mean value of the resource value distribution, while high environmental risk (high variance) means that the actual value may differ significantly from the calculated mean value (can be also much smaller and much larger).

The agents have a memory of their most recent interactions with other agents (last ten interacting agents). The memories record the outcome of the interactions with these other agents and depending on the experience of the agent the probability of the resource sharing action of the agent is altered - it gets more likely to cooperate again with interaction partners who cooperated previously and less likely with those who cheated previously (i.e. the probabilities of the rule components $y, y^{\prime} \rightarrow_{p} t$ and $y, y^{\prime} \rightarrow_{q} h$ change - e.g. the latter gets bigger if the sharing gets more likely according to the past experience).

The agents engage in social learning. They select the individual with the highest amount of resources in their neighborhood as target of imitation - the neighborhood consists of the 10 closest other agents. Two kinds of social learning approaches have been implemented. In one case the agents copy to some extent the communication behavior of the imitated agent by setting their communication rule 
probabilities similar to the matching probabilities of the imitated agent. This is implemented as

$$
\begin{gathered}
p_{\text {revised }}\left(U_{\text {current }}, U^{\prime}{ }_{\text {current }}, U_{\text {new }}\right)=(1-\eta) \cdot p_{\text {original }} \\
\left(U_{\text {current }}, U_{\text {current }}, U_{\text {new }}\right)+\eta \cdot p_{\text {imitated }} \\
\left(U_{\text {current }}, U^{\prime}{ }_{\text {current }}, U_{\text {new }}\right)
\end{gathered}
$$

where $p\left(U_{\text {current }}, U_{\text {current }}, U_{\text {new }}\right)$ is the probability of generating the symbol $U_{\text {new }}$ by the agent after previously having generated the symbol $U_{\text {current }}$ and having received the symbol $U_{\text {current }}^{\prime}$ from the communication partner, and $\eta$ is the extent of the fidelity of the imitation. In the second social learning approach the agent copies fully some of the communication behaviors of the imitated agent. In this case $\eta$, the extent of the fidelity of the imitation, is the probability of copying for all communication rules $L$ (i.e. includes the copying of all related probabilities).

The agents have a limited life span (60 time turns at most in the simulations that are reported here - the agent start their life at a randomly set starting age that is at most 20). When they reach the end of their life they reproduce asexually, by generating potentially mutated offspring which inherit the communication rules with possible small changes to the relevant probabilities. The number of offspring depends on the resources available to the agent $(\rho)$ at the time of death and it is determined by the equation

$$
n=\left[\beta \cdot\left(\left(\rho-\rho_{\text {mean }}\right) / \rho_{\text {stdev }}\right)+\gamma\right]
$$

where $\rho_{\text {mean }}$ and $\rho_{\text {stdev }}$ are the mean and standard deviation of the resource across the whole agent community at the time when the offspring is generated and $\beta$ and $\gamma$ are parameters, [.] is the integer part function $(\beta=1.5, \gamma=1.5)$. We also capped the number of offspring, i.e. if $n>n_{\max }$ then the number of offspring is $n_{\max }\left(n_{\max }=15\right)$. If the above calculation gives $n<1$ then the agent has no offspring.

The offspring of the agent may be spread closely around the location of their parent or may get widely dispersed in the full extent of the two dimensional world in which the agents exist. The first offspring location option may create clumps of cooperating agents, while the second option prevents this. We implemented both options of placing of the offspring of dying agents.

More details about the simulated agent world described above can be found in Andras et al (2003), Andras et al (2006) and Andras (2008). The code developed in Delphi for the implementation of the simulated agent worlds is available on request from the author.

\section{Results and Analysis}

We considered the following six simulation scenarios: (I) partial copying of all rules without wide dispersion of the offspring; (II) partial copying of all rules with wide dispersion of the offspring; (III) full copying of some rules without wide dispersion of the offspring; (IV) full copying of some rules with wide dispersion of the offspring; (V) no social learning and without wide dispersion of offspring; (VI) no social learning and with wide dispersion of offspring. For all scenarios with social learning we considered two variants with low and high levels of copying $(\eta)$, i.e., $\eta=0.2$ and $\eta=0.8$. We ran 20 simulations for five levels of environmental risk ( $\sigma$ $=0.1,0.3,0.5,0.7,0.9$ ) for each variant of the simulation

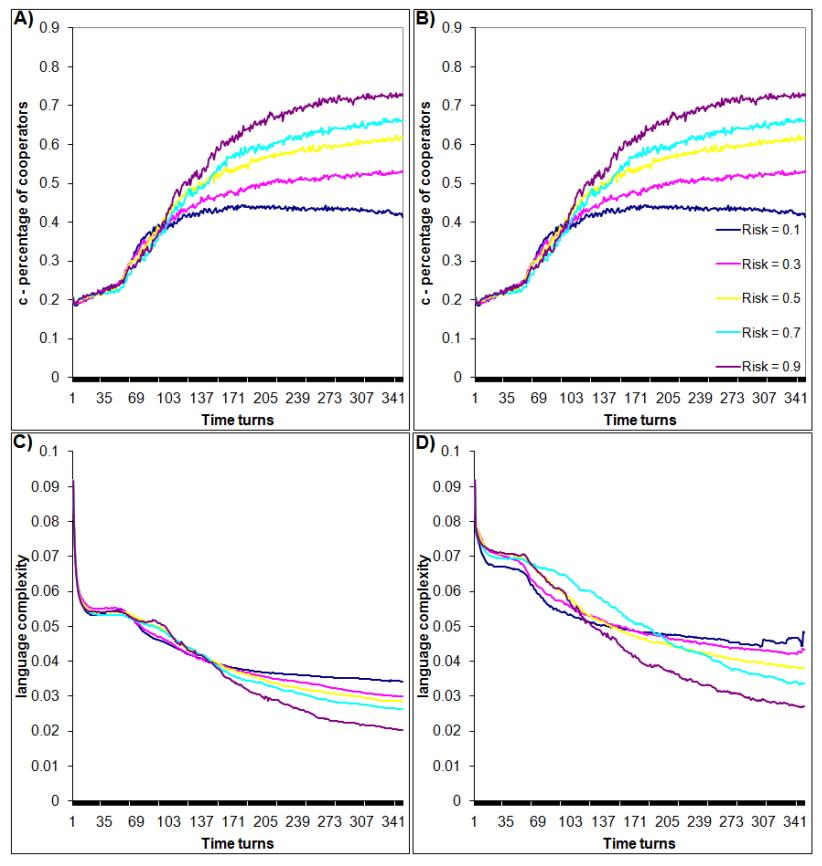

Figure 1. The evolution of the level of cooperation and the level of language complexity for agent communities without wide dispersion of offspring and social learning by partial copying of all language rules (scenario I): A) level of cooperation with $\eta=0.2$; B) level of cooperation with $\eta=$ 0.8 ; C) level of language complexity with $\eta=0.2$; D) level of language complexity with $\eta=0.8$; where $\eta$ is the level of copying.

scenarios. All data shown in the figures are averages of 20 simulation runs, the standard deviations are small and are not shown to not clutter the figures.

In the case of scenarios without wide dispersion of offspring the starting size of the agent population is 1,800 , while in the case of scenarios with wide dispersion of offspring the populations have 7,500 individuals at start. In scenarios with wide dispersal of the offspring the likelihood that an agent dies without offspring is higher than in scenarios with closely located offspring. Thus in scenarios with wide dispersal of the offspring the likelihood that a smaller agent population goes extinct is relatively high. For this reason the population size was increased in these scenarios. Simulations with larger population sizes take more time to run but do not influence the nature of the results presented here.

We measured the level of cooperation $(c)$ by calculating the percentage of agents that engage in a cooperation interaction (i.e. both agents communicate the symbol ' $h$ ' at the end of their interaction) among all agents in the current agent population.

We also measured the complexity of the agent language. For this purpose we considered all language rules $L_{r}, r=$ $1, \ldots, R$ (in the presented agent world simulations we had $R=2$ language rules) and all corresponding probabilities $p_{j, r} j=$ $1, \ldots, k_{r}$ and calculated the variance of the values for each of where $K=\Sigma_{r=1, R} k_{r}$. This language complexity measure is inspired by the concept of Kolmogorov complexity (Li and 

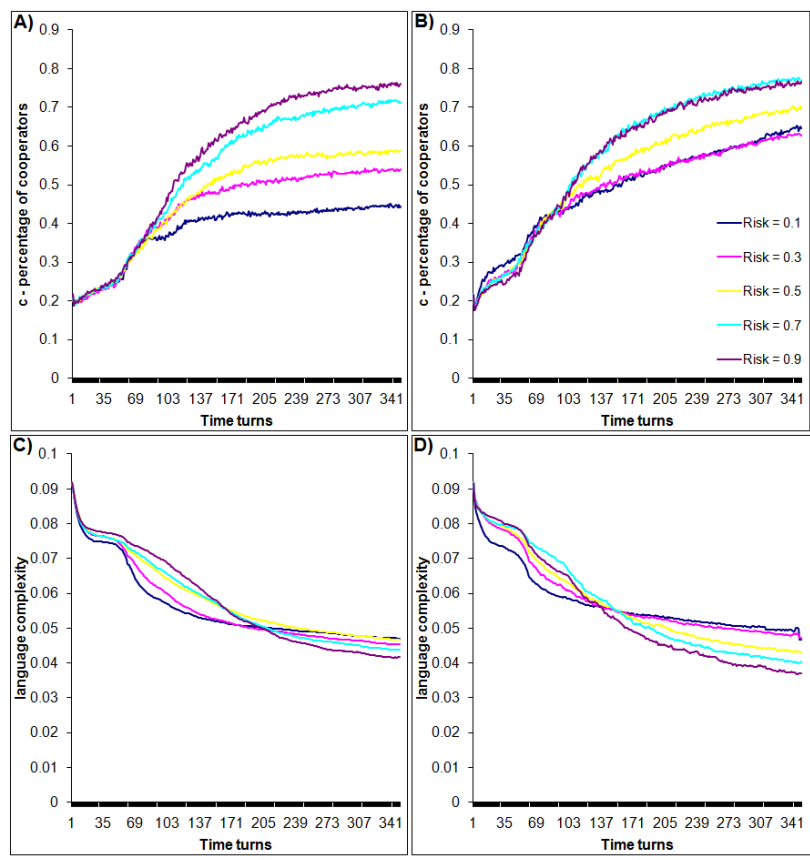

Figure 2. The evolution of the level of cooperation and the level of language complexity for agent communities without wide dispersion of offspring and social learning by full copying of some language rules (scenario III): A) level of cooperation with $\eta=0.2$; B) level of cooperation with $\eta=$ 0.8 ; C) level of language complexity with $\eta=0.2$; D) level of language complexity with $\eta=0.8$; where $\eta$ is the level of copying.

Vitanyi, 1997) in the sense that more variable application of the language rules (higher variance of the corresponding probability values) requires a longer description of the language than the description of a language with the same number of rules but less variable application of the rules.

We expect that allowing the agents to use social learning increases the steady-state level (i.e. after many time turns, when this level gets stabilized) of cooperation in agent communities due to the copying of successful neighboring agents who are expected to be the ones that often cooperate. It is also expected that social learning will reduce the complexity of the language across the agent community, again due to the copying of language rules between agents.

First we considered the scenarios without wide dispersion of the offspring of the agents - scenarios (I), (III) and for reference also scenario (V). For both variants of social learning we analyzed the evolution of the level of cooperation and of the communication complexity for low and high levels of behavioral copying. The results are shown in Figures 1 and 2. These confirm that in both cases of social learning the steady-state level of cooperation grows with the level of environmental risk similar to previously reported results (Andras et al, 2003; Andras et al, 2007). Also, similarly to previous results (Andras, 2008) the results show that the steady-state language complexity decreases with the environmental risk.

Increased level of copying in social learning leads to smaller differences in terms of steady-state levels of

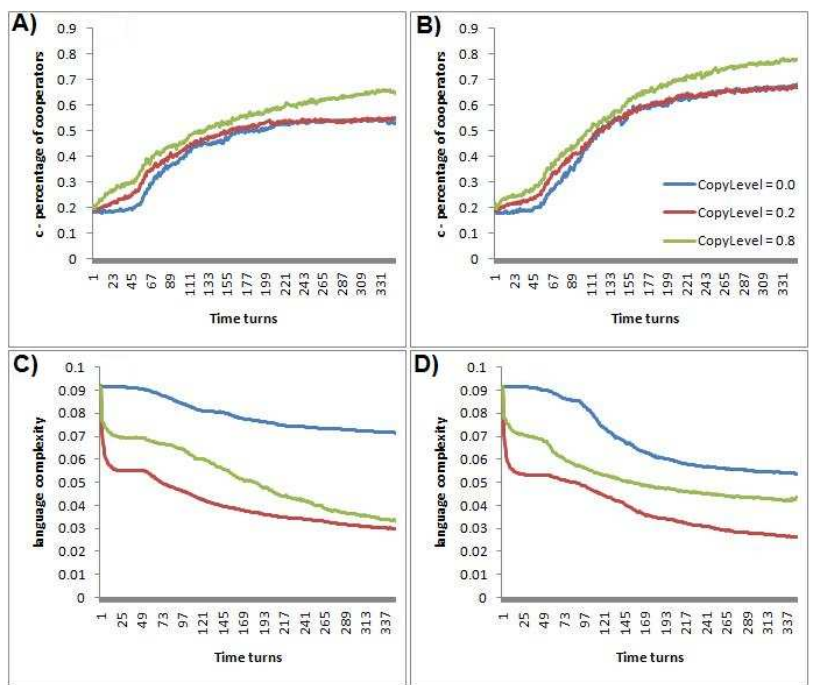

Figure 3. The evolution of the level of cooperation and the level of language complexity for agent communities without wide dispersion of offspring and social learning by partial copying of all language rules (scenarios I and V): A) level of cooperation with $\sigma=0.3$; B) level of cooperation with $\sigma=$ 0.7 ; C) level of language complexity with $\sigma=0.3$; D) level of language complexity with $\sigma=0.7$; where $\sigma$ is the level of environmental risk.

cooperation for different levels of environmental risk, for example the differences for $\sigma=0.7$ and $\sigma=0.9$ become statistically not significantly different for both kinds of
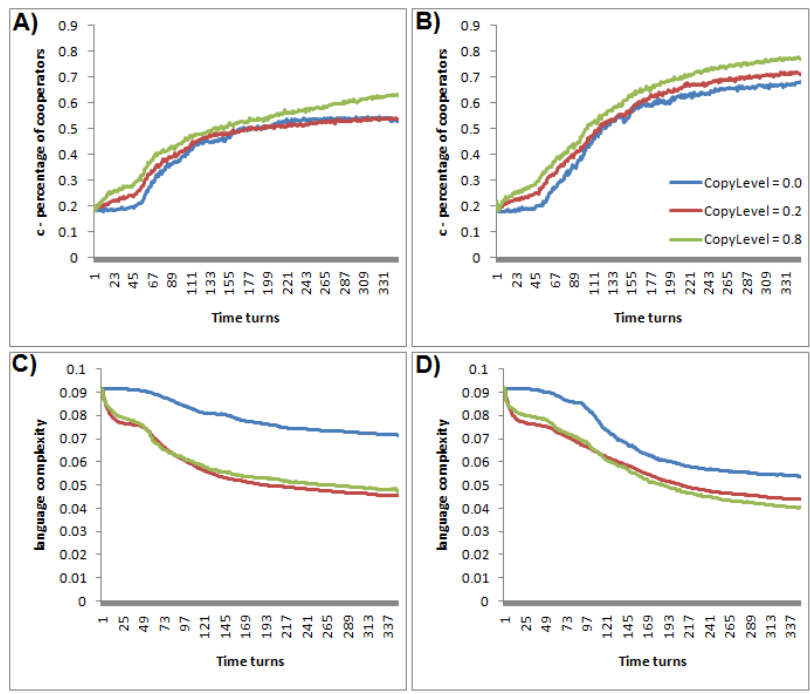

Figure 4. The evolution of the level of cooperation and the level of language complexity for agent communities without wide dispersion of offspring and social learning by full copying of some language rules (scenarios III and V): A) level of cooperation with $\sigma=0.3$; B) level of cooperation with $\sigma=$ 0.7 ; C) level of language complexity with $\sigma=0.3$; D) level of language complexity with $\sigma=0.7$; where $\sigma$ is the level of environmental risk. 


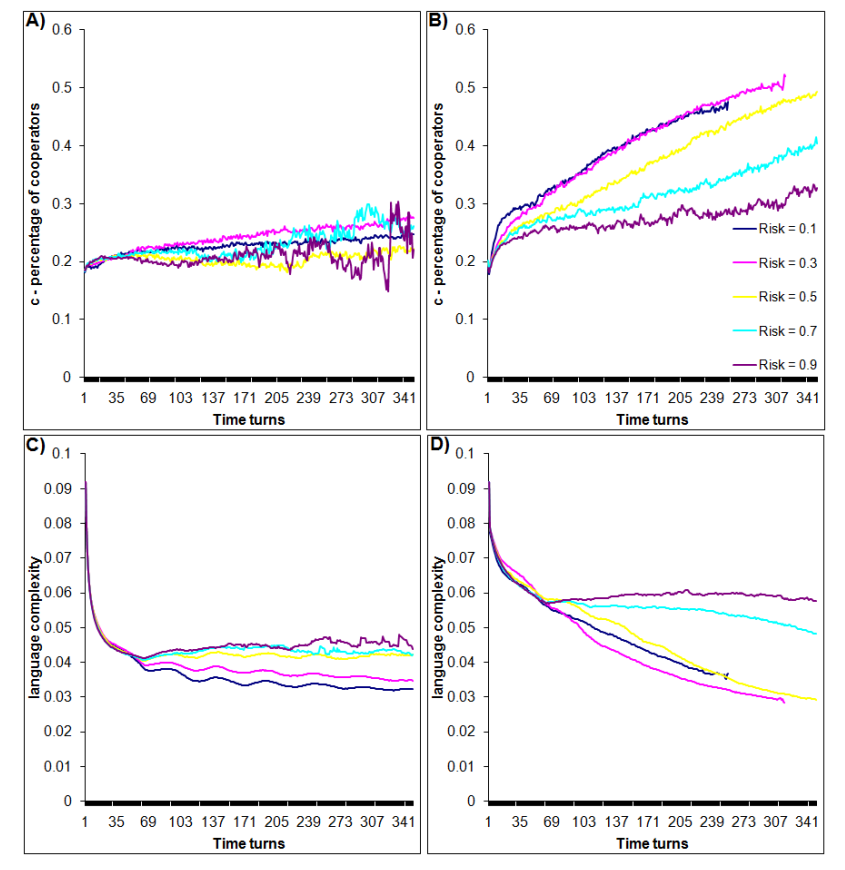

Figure 5. The evolution of the level of cooperation and the level of language complexity for agent communities with wide dispersion of offspring and social learning by partial copying of all language rules (scenario II): A) level of cooperation with $\eta=0.2$; B) level of cooperation with $\eta=0.8$; C) level of language complexity with $\eta=0.2$; D) level of language complexity with $\eta=0.8$; where $\eta$ is the level of copying.

social learning. On the other hand, increased level of copying in social learning leads to increased differences between the steady-state levels of language complexity associated with different levels of environmental risk.

We also note that an impact of the social learning is that at the beginning (until over 120 time turns) the ordering of the language complexity levels associated with risk levels is reversed, i.e. low risk level implies low language complexity. In the absence of social learning the steady-state ordering of risk level associated language complexity levels is already established by around 80 time turns (see Figures 3 and 4). The time point, by which the steady-state ordering of language complexity levels emerges, changes with the level of copying. Interestingly in the case of social learning with partial copying of language rules, higher extent of copying implies delaying this time point, while in the case of social learning with full copying of some rules, the increase in the extent of copying makes this time point earlier.

Next we compared the levels of cooperation and language complexity for different extents of copying in the two kinds of social learning for two fixed levels of environmental risk $\sigma=$ 0.3 and $\sigma=0.7$. The results are shown in Figures 3 and 4 .

The results indicate that social learning at small extent of copying does not change the level of cooperation. However, at lager extent of copying the impact is a statistically significant (t-test, $p=0.05$ ) increase in the level of cooperation. In terms of language complexity both kinds of social learning has a major effect in reducing earlier and by a considerable extent the level of language complexity. Interestingly this effect is

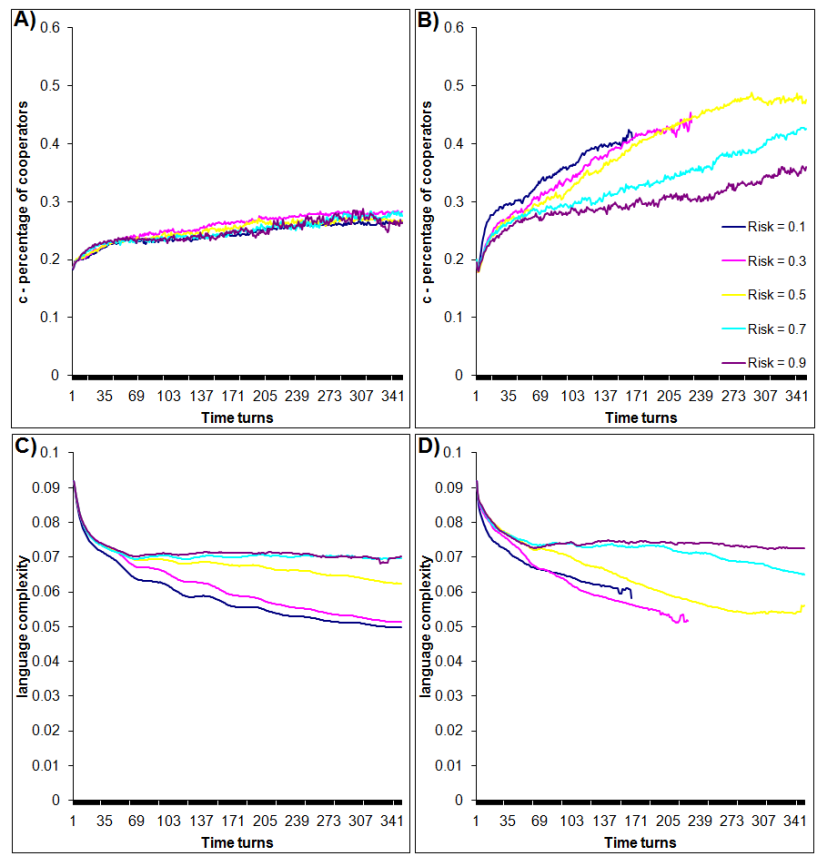

Figure 6. The evolution of the level of cooperation and the level of language complexity for agent communities with wide dispersion of offspring and social learning by full copying of some language rules (scenario IV): A) level of cooperation with $\eta=0.2$; B) level of cooperation with $\eta=0.8$; C) level of language complexity with $\eta=0.2$; D) level of language complexity with $\eta=0.8$; where $\eta$ is the level of copying. The blue and red-purple lines stop early in B) and D) due to the early growth of the simulated populations beyond the population size limit.

larger at lower level of environmental risk and in the case of social learning by partial copying of all language rules the increase in the level of copying reduces the reduction effect on the language complexity.

Next we considered the simulation scenarios with wide dispersion of the offspring - scenarios (II), (IV) and (VI) for reference. The wide dispersion of the offspring reduces in general the level of cooperation in the agent communities, but the ordering of the levels of steady-state cooperation associated with levels of environmental risk remains the same as in the case without wide dispersion of the offspring in the case of agent communities without social learning.

For both kinds of social learning that we implemented we found that the steady-state level of cooperation associated with levels of environmental risk do not follow the ordering pattern found without social learning or with social learning but without wide dispersal of the offspring. In the cases of social learning with wide dispersal of the offspring lower environmental risk leads to higher level of cooperation - the difference becomes statistically significant for higher extent of copying in the social learning. In terms of language complexity again the ordering of the steady-state levels is the reverse of the ordering that we found for scenarios without wide dispersion of the offspring. Lower environmental risk implies higher language complexity in the case of agent societies with widely dispersed offspring and either form of 


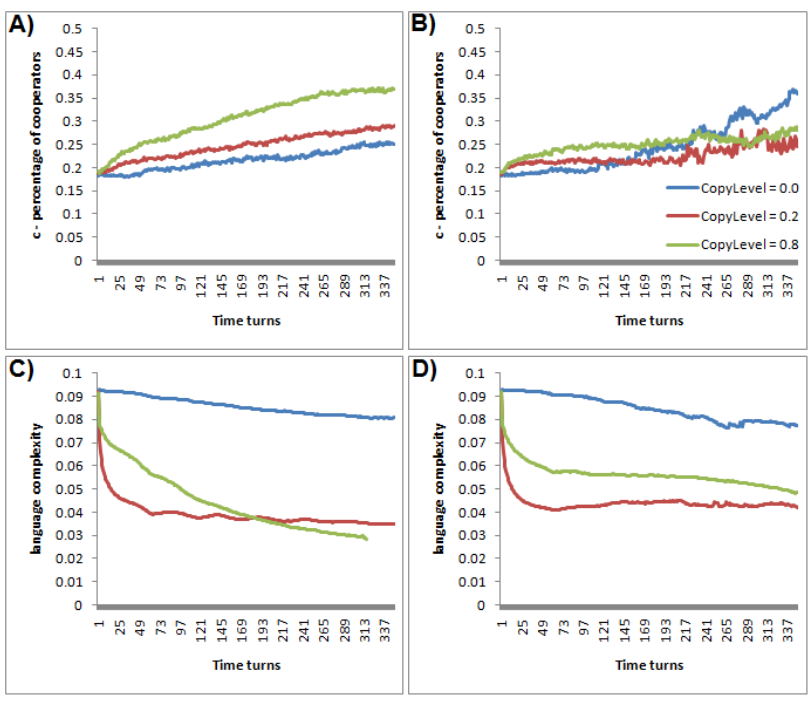

Figure 7. The evolution of the level of cooperation and the level of language complexity for agent communities with wide dispersion of offspring and social learning by partial copying of all language rules (scenarios II and VI): A) level of cooperation with $\sigma=0.3$; B) level of cooperation with $\sigma=$ 0.7 ; C) level of language complexity with $\sigma=0.3$; D) level of language complexity with $\sigma=0.7$; where $\sigma$ is the level of environmental risk.

social learning that we implemented. The results are shown in Figures 5 and 6.

The results show that higher extent of copying in social learning implies an increase in the steady-state level of cooperation for all levels of environmental risk for both kinds of social learning and this effect is stronger in the case of social learning with full copying of some language rules. Similarly, higher extent of copying in social learning increases the effect of environmental risk on the steady-state level of language complexity (i.e. the distinction between steady-state level of language complexity for high and medium level environmental risk becomes clearer). Again, the effect is more accentuated for the social learning with full copying of some language rules. We also note that the steady-state level of language complexity is lower for all levels of environmental risk in the case of social learning with partial copying of all language rules. The evolution of language complexity shows a wavy nature in all cases considered here, which is likely to be due to a generational effect (each generation of agents lasts for around 60 time units).

Further, we considered again two fixed levels of environmental risk $(\sigma=0.3$ and $\sigma=0.7)$ and compared the corresponding levels of cooperation and language complexity for different extents of copying in the two kinds of social learning. The results are presented in Figures 7 and 8.

The results show that at lower level of environmental risk both kinds of social learning increase the level of cooperation relative to the case with no social learning. Notably even at higher levels of environmental risk, at the initial part of the evolution of the agent community the level of cooperation increases with the extent of copying in social learning. For both kinds of social learning, higher extent of copying leads to
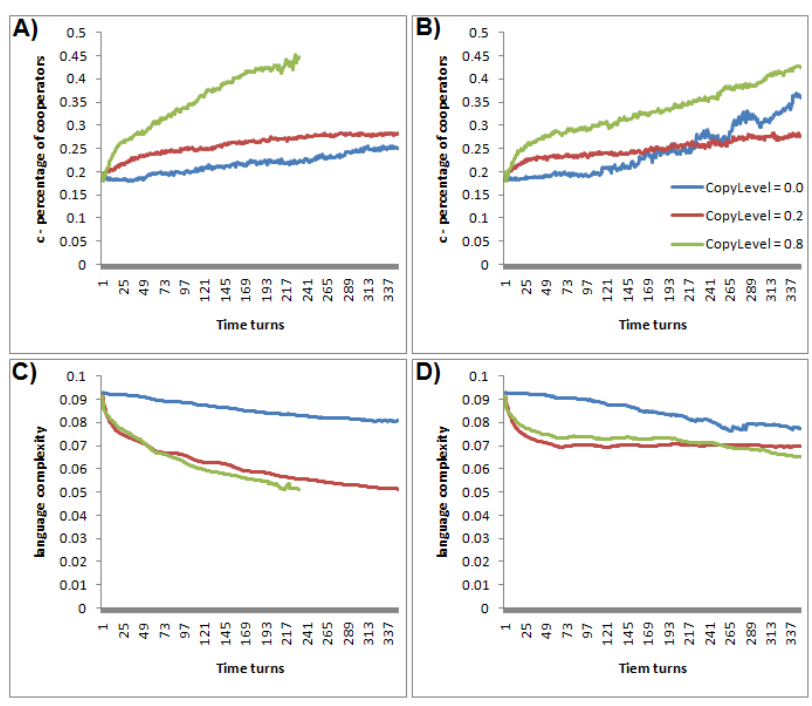

Figure 8. The evolution of the level of cooperation and the level of language complexity for agent communities with wide dispersion of offspring and social learning by full copying of some language rules (scenarios IV and VI): A) level of cooperation with $\sigma=0.3$; B) level of cooperation with $\sigma=$ 0.7 ; C) level of language complexity with $\sigma=0.3$; D) level of language complexity with $\sigma=0.7$; where $\sigma$ is the level of environmental risk. The olive-green line stops early in A) and C) due to the early growth of the simulated populations beyond the population size limit.

higher level of cooperation at both environmental risk levels.

In terms of language complexity, again both kinds of social learning lead to a significant drop in comparison with the case with no social learning. This effect is much larger at the lower level of environmental risk. Higher extent of copying in social learning leads to smaller steady-state language complexity at the lower level environmental risk, at the higher level environmental risk the same effect is smaller. As we already noted, for both kinds of social learning higher level of environmental risk implies higher steady-state language complexity. The level of language complexity is lower for the social learning with partial copying of all language rules than for the social learning with full copying of some language rules for both considered values of extent of copying and for both considered levels of environmental risk.

\section{Discussion and Conclusions}

Our results show that in the simulated agent communities social learning has more effect on the level of cooperation and the level of language complexity at low level environmental risk than at high level of environmental risk. This difference is more accentuated in the case of simulations with wide dispersal of the offspring of agents.

We found that low extent social learning does not increase the level of cooperation and in the case of high environmental risk this may even reduce the level of cooperation. The extent of social learning influences the level of language complexity in all cases. More social learning leads to lower language complexity quicker in the context of low environmental risk. 
A very interesting result is that in the case of simulations with wide dispersal of the offspring adding social learning to the simulations reversed the ordering of levels of cooperation and language complexity associated with levels of environmental risk, compared to the case without social learning. There is no such effect if the offspring of the agents are not dispersed widely in the space where the agents live.

The results suggest that social learning is most impactful in terms of supporting cooperation and reducing language complexity in the context of low environmental risk situations. High environmental risk situations support the emergence of relatively high level of cooperation and low level of language complexity even in the absence of social learning (Krams et al, 2010; Andras et al, 2007; Rand et al, 2014; Potts and Faith, 2015). Thus it is possible that animal or human populations develop high level of cooperation in harsh and risky environments without relying much on social learning, and these populations get to even higher level of cooperation and lower level of language complexity as they move to less harsh and less risky environments.

The results also suggest that social learning gets a much more significant role in communities where related individuals get dispersed widely in the community. In close knit communities where kin are likely to stay close to each other the simulation results suggests that the impact of social learning is mainly in terms of reducing the language complexity within the community.

The observations based on the simulation data that social learning may reduce the level of cooperation or increase the level of language complexity in high risk environments, and that in general it may have little effect on the level of cooperation at small extent of social learning, suggest that social learning has the potential to reduce cooperation in some settings (especially high environmental risk situations). This fits well with some of the experimental observations and theoretical explorations about how social learning may influence negatively the disposition towards cooperation of humans (Molleman et al, 2013; Burton et al, 2015).

In general the results presented here suggest that social learning and environmental risk may take alternating roles in driving animals and humans towards communities that rely increasingly on cooperation among individuals. High environmental risk is the first driver to higher level of cooperation in the community of individuals. Following a move to a low risk environment social learning takes over as driver toward more cooperation and lower language complexity. High level of cooperation in low risk environment combined with social learning may lead to the emergence of novel social structures that add new risks to the environment and also increase the language complexity (Boyce et al, 2012). This may lead to a new high risk environment which in turn facilitates further cooperation in the evolving community. Next, with the maturation of the previously new social structures the environmental risk may get reduced and the community may experience a new bout of increase in cooperation due to social learning. This way the evolving community may increase the level of cooperation and the extent of social institutions, in steps driven alternately by high environmental risk and social learning. The investigation of generation of novel social structures in agent-based simulations of communities will be part of future work.

\section{References}

Andras, P. (2008). Uncertainty and communication complexity in iterated cooperation games, in Proceedings of ALife XI, pp.9-16.

Andras, P., Lazarus, J., Roberts, G., and Lynden, S. J. (2006). Uncertainty and cooperation: Analytical results and a simulated agent society. JASSS - Journal of Artificial Societies and Social Simulation, 9:1/7.

Andras, P., Lazarus, J., Roberts, G. (2007). Environmental adversity and uncertainty favour cooperation. BMC Evolutionary Biology, 7:240.

Andras, P., Roberts, G., and Lazarus, J. (2003). Environmental risk, cooperation and communication complexity. In Alonso, E., Kudenko, D., and Kazakov, D., editors, Adaptive Agents and MultiAgent Systems, pages 49-65. Springer-Verlag, Berlin.

Axelrod, R. (1997). The Complexity of Cooperation: Agent-Based Models of Competition and Collaboration. Princeton University Press, Princeton, NJ.

Boyce, W.T., Sokolowski, M.B. and Robinson, G.E. (2012). Toward a new biology of social adversity, PNAS, 109:17143-17148.

Boyd, R. and Richerson, P.J. (2009). Culture and the evolution of human cooperation. Philosophical Transactions of the Royal Society B, 364:3281-3288

Burton-Chellew, M.N., Nax, H.H., and West, S.A. (2015). Payoff-based learning explains the decline in ccoperation in public good games. Proceedings of the Royal Society B, 282:20142678.

Chudek, M., Zhao, W. and Henrich, J. (2013). Culture-gene coevolution, large-scale cooperation and the shaping of human social psychology. In: Sterelny, K., Joyce, R., Calcott, B. and Fraser, B (eds.) Cooperation and Its Evolution, MIT Press, pp.425-458.

Flinn, M.V. (1997). Culture and the evolution of social learning. Evolution and Human Behavior, 18:23-67.

Heyes, C. (2013). What can imitation do for cooperation? In: Sterelny, K., Joyce, R., Calcott, B. and Fraser, B (eds.) Cooperation and Its Evolution, MIT Press, pp.313-332.

Krams, I., Krama, T., Berzins, A. and Rantala, M.J. (2010). The risk of predation favors cooperation among breeding prey. Communicative \& Integrative Biology, 3:243-244.

Li, M. and Vitanyi, P. M. B. (1997). An Introduction to Kolmogorov Complexity and its Applications. Springer, New York.

Molleman, L., Pen, I. and Weissing, F.J. (2013). Effects of conformism on the cultural evolution of coial behavior. PLOS ONE, 8:e68153.

Nakahashi, W., Wakano, J.Y. and Henrich, J. (2012). Adaptive social learning strategies in temporally and spatially varying environments. Human Nature, 23:386-418.

Potts, R. and Faith, J.T. (2015). Alternating high and low climate variability: The context of natural selection and speciation in PlioPleistocene hominin evolution. Journal of Human Evolution, 87:520.

Rand, D.G. and Nowak, M. (2013). Human cooperation. Trends in Cognitive Sciences, 17:413-425.

Rand, D.G., Peysakhovich, A., Kraft-Todd, G.T., Newman, G.E., Wurzbacher, O., Nowak, M.A. and Greene, J.D. (2013). Social heuristics shape intuitive cooperation. Nature Communications, 5:3677.

Rendell, L., Boyd, R., Cownden, D., Enquist, M., Eriksson, K., Feldman, M.W., Fogarty, L., Ghirlanda, S., Lillicrap, T. and Laland, K.N. (2010). Why copy others? Insights from the social learning strategies tournament. Science, 328:208-213.

Seltzer, N. and Smirnov, O. (2015). Degrees of separation, social learning, and the evolution of cooperation in a small-world network. Journal of Artificial Societies and Social Simulation, 18:12.

Sigmund, K., De Silva, H., Traulsen, A. and Hauert, C. (2010). Social learning promotes institutions for governing the commons. Nature, 466:861-863.

Spinks, A. C., Jarvis, J. U. M., and Bennett, N. C. (2000). Comparative patterns of philopatry and dispersal in two common mole-rat populations: implications for the evolution of mole-rat sociality. Journal of Animal Ecology, 69:224-234. 